\title{
Dark Matter Distributions of the Milky Way Satellites and Implications for Indirect Detection
}

\section{Louis E. Strigari*}

Kavli Institue for Particle Astrophysics and Cosmology, Physics Department, Stanford University, Stanford, CA 94305

E-mail: strigariestanford.edu

\section{Gregory D. Martinez}

Center for Cosmology, Department of Physics and Astronomy, University of California-Irvine, Irvine, CA 92697

E-mail: gmartine@uci.edu

\begin{abstract}
We review recent progress in understanding the mass distributions of the Milky Way dwarf satellite population, with a specific focus on the implications for gamma-ray fluxes from dark matter. We outline a method that self-consitently predicts the gamma-ray fluxes from the satellites using line-of-sight velocities, the Constrained Minimal Supersymmetric Standard Model (CMSSM), and a model for the boost from substructure in the dark matter halos. Combining all of these ingredients, and accounting for the reduced astrophysical backgrounds relative to the Galactic center, the dwarf satellites make strong candidates for indirect detection with Fermi/GLAST and future Air Cerenkov Telescopes.
\end{abstract}

Identification of dark matter 2008

August 18-22, 2008

Stockholm, Sweden

\footnotetext{
*Speaker.
} 


\section{Introduction}

The Milky Way has at least two dozen known satellite galaxies with observed luminosities that vary in the range from $\sim 10^{3}-10^{7} \mathrm{~L}_{\odot}$. Eleven of these satellites were discovered before 2004 [1], while the remaining satellites have been discovered since then by the Sloan Digital Sky Survey (SDSS) [2]. The pre-2004 classical satellites all have half-light radii of a few hundreds of parsecs, which is one of the classical lines of evidence that these objects are distinct from Galactic globular clusters, which have half-light radii no more than about 10 parsecs [3]. However, despite this difference in their half-light radii, globular clusters and dwarf satellite galaxies share common average velocity dispersions of $\sim 5-15 \mathrm{~km} / \mathrm{s}$. Globular clusters with this range of velocity dispersions have total mass-to-light ratios of order unity within their observed stellar radii. The more spatially-extended dwarf satellites, on the other hand, are found to have mass-to-light ratios within their stellar extent $\sim 10-100$, implying that these objects are strongly dark matter dominated.

The above distinction between dwarf galaxies and globular clusters has been challenged recently by the discovery of several extremely low luminosity $\left(\sim 10^{3} \mathrm{~L}_{\odot}\right)$ satellites that have halflight radii of $\sim 20-50 \mathrm{pc}$. Are these objects closer in nature to the globular clusters, or the dark matter-dominated dwarf satellites? Kinematics studies of these extreme objects suggest that many are consistent with interpretation as dwarf galaxies, making them the least luminous and most dark matter dominated galaxies known [5, 6]. However, in other cases, it is still unclear if tidal disruption is playing a role in shaping the dynamics (Willman et al. in prep).

The presence of a population of dark matter dominated objects in close proximity to the Milky Way provides an important test of our understanding of galaxy formation at the smallest scales. Also, because of their high mass-to-light ratios, the Milky Way satellites make ideal laboratories for testing the properties of the dark matter that dominates the mass of these objects. In the remaining sections, we will review evidence that makes these satellites the most dark matter-dominated objects known, and then focus on the specific application of indirect detection from particle dark matter annihilation in these halos.

\section{Dark Matter Distributions}

Thanks to high resolution spectra, the past few years have seen a great increase in the number of line-of-sight velocities for stars in dwarf satellites [5, 7]. For the classical satellites, the velocity dispersions are observed to remain flat out to the stellar tidal radius, which implies that massfollows-light models, which assume that all of the mass distribution is accounted for by the stars, are conclusively ruled out. To obtain a more accurate assessment of the mass distribution of the satellites, two components models are thus required, which separately account for the distribution of stars and dark matter.

Given the large data sets now available, it is important to ask, in the context of two-component model, which physical quantities are best constrained by the line-of-sight velocity data. Answering this question first requires defining a set of model parameters. The dark matter halos typically are described by several free parameters: these include a scale density, a scale radius, an asymptotic inner and outer slope, a transition between these latter two slopes. Additionally the velocity anisotropy of the stars is unknown, and it is even possible that this latter quantity varies with 
physical radius in the galaxy and is described by several free parameters. However, despite the uncertainties in all of these parameters, quantities such as the integrated mass within the half-light radius are strongly constrained by current data samples [4].

In order to facilitate a comparison to the mass function of subhalos in Cold Dark Matter (CDM) simulations, it is useful to determine the mass of the dwarf satellites within a fixed physical radius; here we quote mass results within 300 and 600 pc. The former represents an average of the halflight radii of the entire satellite population, while the latter represents an average half-light radius for only the classical population of pre-SDSS satellites. Further, the mass within 600 pc may be ill-defined for several of the new satellites, as this radius is beyond the present value of the Jacobi radius [6]. Accounting for the model uncertainties discussed above, the masses of the classical dwarf satellites within a radius of $600 \mathrm{pc}$ are found to fall within the range of $\sim[1-6] \times 10^{7}$ $M_{\odot}[8,7]$. However, the range of masses within $300 \mathrm{pc}$ is much more narrow: all of the satellites have a mass very close to $10^{7} \mathrm{M}_{\odot}$ within $300 \mathrm{pc}$, in spite of the nearly five order of magnitude spread in the luminosities of the satellites [9].

The fact that all of the dwarf satellites share a common mass scale of $10^{7} \mathrm{M}_{\odot}$ within $300 \mathrm{pc}$ provides clues into either the formation of galaxies at small scales, the nature of the particle dark matter, or perhaps both. Is this result of a common mass scale consistent with CDM simulations? Although no currently published numerical simulations are able to resolve the mass of subhalos within $300 \mathrm{pc}$ from their centers, initial results that extrapolate the subhalo mass distributions from larger radii show that this large luminosity scatter at fixed subhalo mass can be explained by understanding the distribution of halo masses upon accretion into the Milky Way [10].

\section{Indirect Detection: Predicted Gamma-Ray Fluxes}

Given their high mass-to-light ratios and their proximity to the Sun dwarf satellites also make interesting objects to search for the presence of particle dark matter annihilation. Considering specifically the case of annihilation to gamma-rays, although the mean value of the dark matter annihilation flux from these satellites is probably smaller than the flux from the Galactic center region, dwarf satellites are unique targets because 1) the astrophysical gamma-ray backgrounds towards these objects are much smaller than the backgrounds in the region of the Galactic center, and 2) the dark matter distributions are much more strongly constrained than the dark matter distribution near the Galactic center. In the CDM model, the central density slopes of the dark matter-dominated dwarf satellites should be very close to the $1 / r$ scaling seen in numerical simulations; a central slope near this value is much less certain in the Galactic center because of the uncertain effects of the baryons in shaping the halo profile.

There have been many previous studies that have examined the prospects for indirect detection from dwarf satellites [11]. These studies, which were undertaken primarily before the era of high resolution spectroscopy and large data sets from dwarf satellites, generally found that the uncertainty in the contribution to the flux from dark matter distributions implied orders of magnitude uncertainty in the flux from these objects. However, with the modeling for the mass distribution of these halos as described above, and assuming the CDM model, the astrophysical uncertainty in the flux is now much more strongly constrained [12]. We are now at the point where assessment of the best targets, or sets of targets, is now feasible. Since all of the satellites have roughly the same 
mass in dark matter within the region where most of the gamma-ray emission comes from, the most important factor in determining detectability is the distance to each object and the non-dark matter gamma-ray backgrounds in the direction of each object [14]. Interestingly, the nearest of the satellites are also the most dark matter-dominated, and all objects have strong limits on their gas content [13], a fact that ultimately may help the detection of a dark matter signal.

When determining the contribution to the gamma-ray flux from the dark matter distributions, it is important to quantify the boost factor from halo substructure. Since the gamma-ray flux is proportional to the halo density profile squared, the presence of substructure will act to enhance the flux, though the degree of this enhancement is poorly constrained. Strigari et al. [12] showed that, under optimistic conditions for the density profiles for the substructures (and sub-sub structures), there is an upper limit to the boost factor of about 100. Recent numerical simulations are now reaching the resolution in which a direct calculation of the boost factor is possible, at least for substructures greater than about $10^{4} \mathrm{M}_{\odot}[15,16]$.

Understanding the structure of the dark matter halos of the dwarf satellites provides only half of the story when calculating the gamma-ray flux. To self-consistently calculate the flux, we must specify the particle dark matter model. In this work, we will consider Supersymmetry (SUSY), which provides a compelling and well-motivated extension to the Standard Model that naturally contains a dark matter candidate. Since SUSY is characterized by a large number of free parameters, one often considers simplification to this model which requires SUSY parameter unification at grand unification (GUT) scales. This so-called constained MSSM (CMSSM) now limits the number of SUSY parameters to five: the common gaugino mass $\left(m_{1 / 2}\right)$, scalar mass $\left(m_{0}\right)$, trilinear scalar couplings $\left(A_{0}\right)$, the ratio of the higgs expection values $(\tan \beta)$, and the sign of the " $\mu$ term" $(\operatorname{sgn}(\mu))$. SuperBayes, a MCMC software package developed by Ruiz de Austri et al. [17] to explore CMSSM parameter space, uses a suite of programs to derive various CMSSM parameters, given experimental constraints. For the results presented here, we use nine converged MCMC chains of approximately 30 thousand points each, kindly provided to us by R. Trotta.

In Figure 1, we present the results of an MCMC scan of the parameter space of both the astrophysical and particle physics components to the flux, showing the likelihood for the flux versus the annihilation cross section. We show two satellites: Segue 1 and Draco. The mass of Segue 1 has been determined recently using the spectra of 24 stars, with all current lines of evidence suggesting that this object is the most dark-matter dominated galaxy known [6]. Draco is a classical Milky Way satellite, with a mass profile based on the kinematics of several hundred stars [7]. Given these fluxes, these satellites make interesting targets for study with gamma-ray satellites such as Fermi/GLAST [19] and future Air Cerenkov telescopes [20]

\section{Conclusion}

Milky Way satellites are ideal for testing the nature of dark matter on small scales. Here we have reviewed the calculation of their mass distribution, and examined the implications for indirect detection of dark matter annihilation. We have reviewed a framework that self-consistently calculated the gamma-ray fluxes, given constraints from both the CMSSM and the kinematics of stars in the satellites. Depending on the boost factor from halo substructure and the properties of the dark matter particle, the gamma-ray flux from these satellites may be resolvable via Fermi/GLAST 

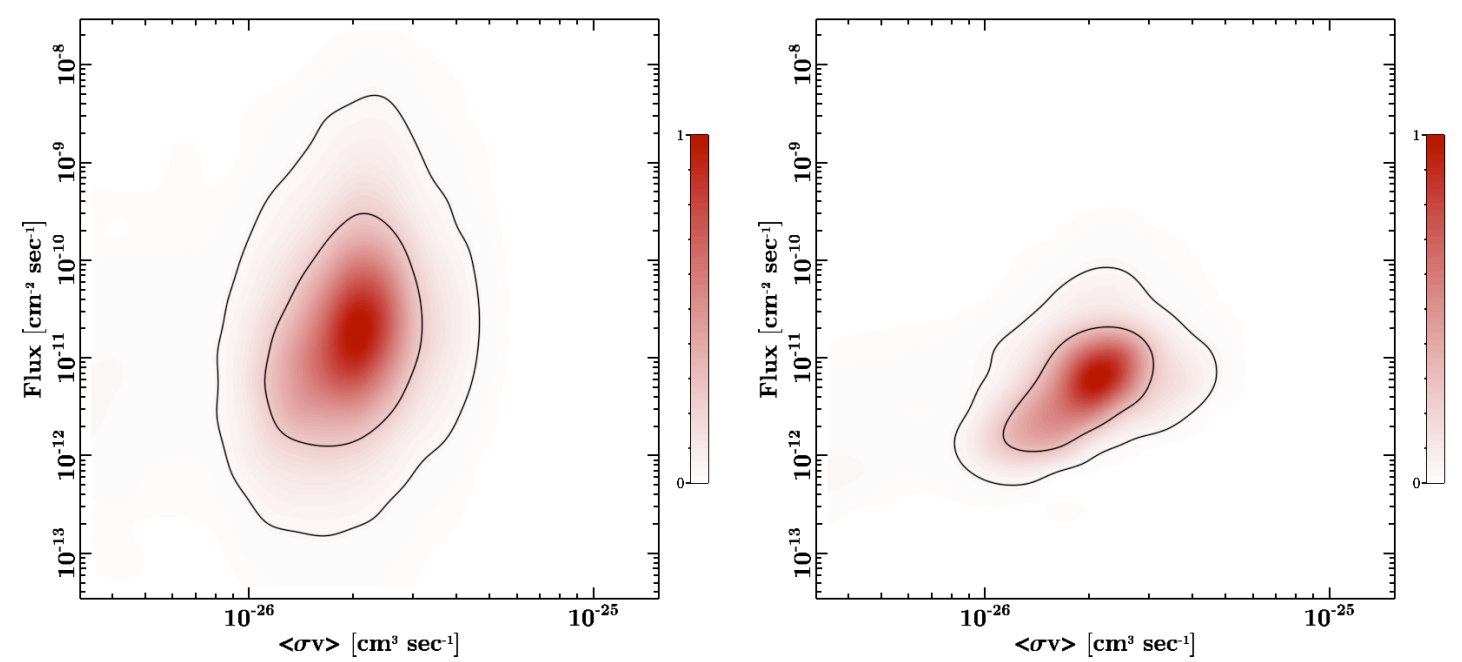

Figure 1: The predicted gamma-ray flux, for energies $>1 \mathrm{GeV}$, from Segue 1 (left), and Draco (right) assuming the Constrained Minimal Supersymmetric Standard Model (CMSM). We self-consistently marginalize over the dark matter distributions using the line-of-sight velocities and calculate the boost factor from halo substructure given a CMSSM model and using the subhalo model described in Bullock et al. [18]. Inner and outer contours represent the $68 \%$ and $95 \%$ c.l. region. Figure from Martinez et al., 2009.

and future Air Cerenkov Telescopes. Although the present census of Milky Way satellites lies at about two dozen, given incompleteness limits there are likely many more satellites to be discovered [21]. Classifying the dark matter distributions of these objects will provide important future constraints on small scale galaxy formation, and add many more targets to search for particle dark matter annihilation.

\section{Acknowledgements}

We thank James Bullock, Marla Geha, Manoj Kaplinghat, Savvas Koushiappas, Josh Simon, Erik Tollerud, Matt Walker, Beth Willman, and Joe Wolf, for collaborations during the course of this work. Support for this L.E.S. was provided by NASA through Hubble Fellowship grant HF01225.01 awarded by the Space Telescope Science Institute, which is operated by the Association of Universities for Research in Astronomy, Inc., for NASA, under contract NAS 5-26555.

\section{References}

[1] M. Mateo, Ann. Rev. Astron. Astrophys. 36, 435 (1998) [arXiv:astro-ph/9810070].

[2] B. Willman et al., Astrophys. J. 626, L85 (2005) [arXiv:astro-ph/0503552]; D. B. Zucker et al. [SDSS Collaboration], Astrophys. J. 643, L103 (2006) [arXiv:astro-ph/0604354]; D. B. Zucker et al., Astrophys. J. 650, L41 (2006) [arXiv:astro-ph/0606633]; V. Belokurov et al. [SDSS Collaboration], Astrophys. J. 654, 897 (2007) [arXiv:astro-ph/0608448]; S. M. Walsh, H. Jerjen, \& B. Willman, Astrophys. J. 662, L83 (2007) arXiv:0705.1378 [astro-ph]; V. Belokurov et al., arXiv:0807.2831 [astro-ph]. 
[3] G. Gilmore, M. I. Wilkinson, R. F. G. Wyse, J. T. Kleyna, A. Koch, N. W. Evans and E. K. Grebel, Astrophys. J. 663, 948 (2007) [arXiv:astro-ph/0703308].

[4] L. E. Strigari, J. S. Bullock and M. Kaplinghat, Astrophys. J. 657, L1 (2007) [arXiv:astro-ph/0701581].

[5] J. D. Simon and M. Geha, Astrophys. J. 670, 313 (2007) [arXiv:0706.0516 [astro-ph]].

[6] M. Geha, B. Willman, J. D. Simon, L. E. Strigari, E. N. Kirby, D. R. Law and J. Strader, arXiv:0809.2781 [astro-ph].

[7] M. G. Walker, M. Mateo, E. W. Olszewski, O. Y. Gnedin, X. Wang, B. Sen and M. Woodroofe, Astrophys. J. 667, L53 (2007) arXiv:0708.0010 [astro-ph].

[8] L. E. Strigari, J. S. Bullock, M. Kaplinghat, J. Diemand, M. Kuhlen and P. Madau, Astrophys. J. 669, 676 (2007) [arXiv:0704.1817 [astro-ph]].

[9] L. E. Strigari, J. S. Bullock, M. Kaplinghat, J. D. Simon, M. Geha, B. Willman and M. G. Walker, Nature 454, 1096 (2008) [arXiv:0808.3772 [astro-ph]].

[10] Y. S. Li, A. Helmi, G. De Lucia and F. Stoehr, arXiv:0810.1297 [astro-ph]; A. V. Maccio’, X. Kang and B. Moore, arXiv:0810.1734 [astro-ph].

[11] E. A. Baltz, C. Briot, P. Salati, R. Taillet and J. Silk, Phys. Rev. D 61, 023514 (2000) [arXiv:astro-ph/9909112]; C. Tyler, Phys. Rev. D 66, 023509 (2002) [arXiv:astro-ph/0203242]; N. W. Evans, F. Ferrer and S. Sarkar, Phys. Rev. D 69, 123501 (2004) [arXiv:astro-ph/0311145]; L. Bergstrom and D. Hooper, Phys. Rev. D 73, 063510 (2006) [arXiv:hep-ph/0512317]; S. Profumo and M. Kamionkowski, JCAP 0603, 003 (2006) [arXiv:astro-ph/0601249]; T. Bringmann, M. Doro and M. Fornasa, arXiv:0809.2269 [astro-ph].

[12] L. E. Strigari, S. M. Koushiappas, J. S. Bullock and M. Kaplinghat, Phys. Rev. D 75, 083526 (2007) [arXiv:astro-ph/0611925].

[13] M. E. Putman, J. Grcevich and J. E. G. Peek, arXiv:0803.3069 [astro-ph].

[14] L. E. Strigari, S. M. Koushiappas, J. S. Bullock, M. Kaplinghat, J. D. Simon, M. Geha and B. Willman, Astrophys. J. 678, 614 (2008) [ arXiv:0709.1510 [astro-ph]].

[15] M. Kuhlen, J. Diemand and P. Madau, Astrophys. J. 686, 262 (2008) [arXiv:0805.4416 [astro-ph]].

[16] V. Springel et al., arXiv:0809.0894 [astro-ph].

[17] R. R. de Austri, R. Trotta and L. Roszkowski, JHEP 0605, 002 (2006) [arXiv:hep-ph/0602028].

[18] J. S. Bullock et al., Mon. Not. Roy. Astron. Soc. 321, 559 (2001) [arXiv:astro-ph/9908159].

[19] E. A. Baltz et al., JCAP 0807, 013 (2008) [arXiv:0806.2911 [astro-ph]].

[20] C. M. Hui and f. t. V. Collaboration, arXiv:0810.1913 [astro-ph]; E. Aliu et al. [MAGIC Collaboration], arXiv:0810.3561 [astro-ph].

[21] S. Koposov et al., Astrophys. J. 686, 279 (2008) [arXiv:0706.2687 [astro-ph]]; E. J. Tollerud, J. S. Bullock, L. E. Strigari and B. Willman, arXiv:0806.4381 [astro-ph]; S. Walsh, B. Willman and H. Jerjen, arXiv:0807.3345 [astro-ph]. 